\title{
Amorphous and Nano-Crystalline Materials in Pristine Carbonaceous Chondrite Meteorites
}

Neyda M. Abreu ${ }^{1, *}$, C. M. Corrigan ${ }^{2}$, D. C. Hezel ${ }^{3}$, L. P. Keller ${ }^{4}$, K. T. Howard ${ }^{5}$, B. T. De Gregorio ${ }^{6}$, K. L. Crispin ${ }^{1}$, J. Gray ${ }^{1}$, H. Wang ${ }^{1}$

1. Penn State Univ., PA, 15801, USA.

2. Smithsonian Institution, Washington, DC, USA.

3. Dept. of Geology and Mineralogy, Univ. of Cologne, Germany.

4. ARES, NASA/JSC, Houston, TX, USA.

5. Earth and Environmental Sci., CUNY Graduate Center, New York, NY, 10016, USA.

${ }^{6 .}$ U.S. Naval Research Laboratory, Washington, DC, USA.

* Corresponding author: abreu@psu.edu

Carbonaceous chondrites (CCs) are meteorites that come from asteroids that never fully melted and represent materials formed and processed $\sim 4.56 \mathrm{Ga}$. CCs are made up of: (1) matrices dominated by sub- $\mu \mathrm{m}$ phases; (2) chondrules, containing silicate phenocrysts, mesostasis, and Fe-Ni metal; and (3) Ca- Al-rich inclusions. Imposed on these materials is a variable history of impacts, thermal metamorphism, and hydrothermal alteration specific to the source asteroid. Understanding how asteroidal processes change the physical, compositional, and spectral characteristics of CCs is a fundamental step in their exploration. Here, we study the most susceptible materials in the most pristine CCs - including nanophase silicates and feldspathic mesostases. We characterized these materials in some of the most pristine CCs, belonging to the CR and CM subgroups.

Here, we present analyses collected using: Position sensitive detector X-ray diffraction; SEM (FEI Quanta 200 operating at 20kV); EMPA (JEOL 8530F, Cameca SXFive, and Cameca sx-100); TEM (JEOL 2200FS and Talos F200X). FIB sections for TEM were prepared using a Helios Quanta NanoLab 660. Analytical studies were performed at the Materials Characterization Lab at Penn State, Smithsonian Institution, Kingsborough CUNY, U.S. Naval Research Laboratory, and at NASA Johnson Space Center.

Matrices in Pristine CR chondrites: 9 Antarctic CRs were analyzed (EET 96259, GRA 95229, GRO 95577, GRO 03116, LAP 02342, LAP 04516, LAP 04720, MIL 07525, and MIL 090001) [e.g. 1]. Based on PDS-XRD and TEM, amorphous and crystalline Fe-Mg silicates are the dominant phases in CRs. Amorphous silicates are progressively replaced by phyllosilicates (Fig. 1a-d). FIB-TEM observations show nano-phase $\mathrm{Fe}(\mathrm{Ni})$-sulfides and varying amounts of Fe-oxides, and $\mathrm{Ca}$-carbonates are also present. The relative abundances of amorphous Fe-Mg silicates to phyllosilicates decrease as the abundances of Fe-oxides and the oxidation of Fe-Ni sulfides increase. The mineral assemblages in the matrices become more variable and heterogeneous as new and coarser phyllosilicates are formed.

Mesostasis in Pristine CR chondrites: We studied chondrule mesostases in the same CRs and found that their degree of alteration was consistent with the characteristics of matrices. Mesostases in weakly altered CRs are pristine, as evidenced by high abundances of primary crystalline nanophases, minor residual glass, distinct compositional differences depending on chondrule composition, and absence of trends attributable to element mobilization (Fig. 1e-i). Our TEM observations challenge the longaccepted assumption that pristine carbonaceous chondrites contain a glassy mesostasis. We observed 
abundant nanophase crystalline pyroxene, plagioclase, and olivine - the presence of minerals probably makes them less susceptible to asteroidal processes than previously thought. Mobilization trends that had been previously suggested for mesostases in CRs and other CCs [e.g., 2-3] were not observed. Replacement of interchondrule materials by phyllosilicates is only prevalent in extensively aqueously altered CRs.

Matrices in Pristine CM chondrites: Despite numerous studies of the mineralogy and petrology of CMs [e.g. 4-6], mildly altered CMs are scarce. We are only starting to learn about the primary characteristics of the matrices in the most pristine CMs. We identified mildly-altered Antarctic CMs, LAP 04514, LAP 04527, LAP 04565, and LAP 02333 (i.e., LAP CMs). Based on the presence of Fe-Mg amorphous silicates, abundances of nanophase Fe-Ni sulfides, the composition of tochilinite, and carbonaceous materials are dominated by nanoglobules, the LAP CMs show affinities with the least altered CMs, such as the Paris meteorite [6,7]. The matrix in the LAP CMs shows a greater degree of hydrothermal alteration than pristine CRs. We did not observe evidence of thermal metamorphism in the fine-grained matrix materials, such as the collapse of phyllosilicate crystal structure and $\mathrm{S}$ and $\mathrm{Na}$ losses.

\section{References:}

[1] N. M. Abreu. Geochimica et Cosmochimica Acta 194 (2016) p. 91-122.

[2] P. V. Burger M.S. thesis, University of New Mexico, Albuquerque, NM, USA (2005).

[3] J. Grossman and A. J. Brearley, Meteoritics \& Planetary Sciences 40 (2005) p. 87-122.

[4] A. Rubin et al., Geochimica et Cosmochimica Acta 71 (2007) p. 2361-2382.

[5] K. Metzler et al., Geochimica et Cosmochimica Acta 56 (1992) p. 2873-2897.

[6] R. H. Hewins et al., Geochimica et Cosmochimica Acta 124 (2014) p. 190-222.

[7] H. G. Changela et al., Geochimica et Cosmochimica Acta 159 (2015) p. 285-297.
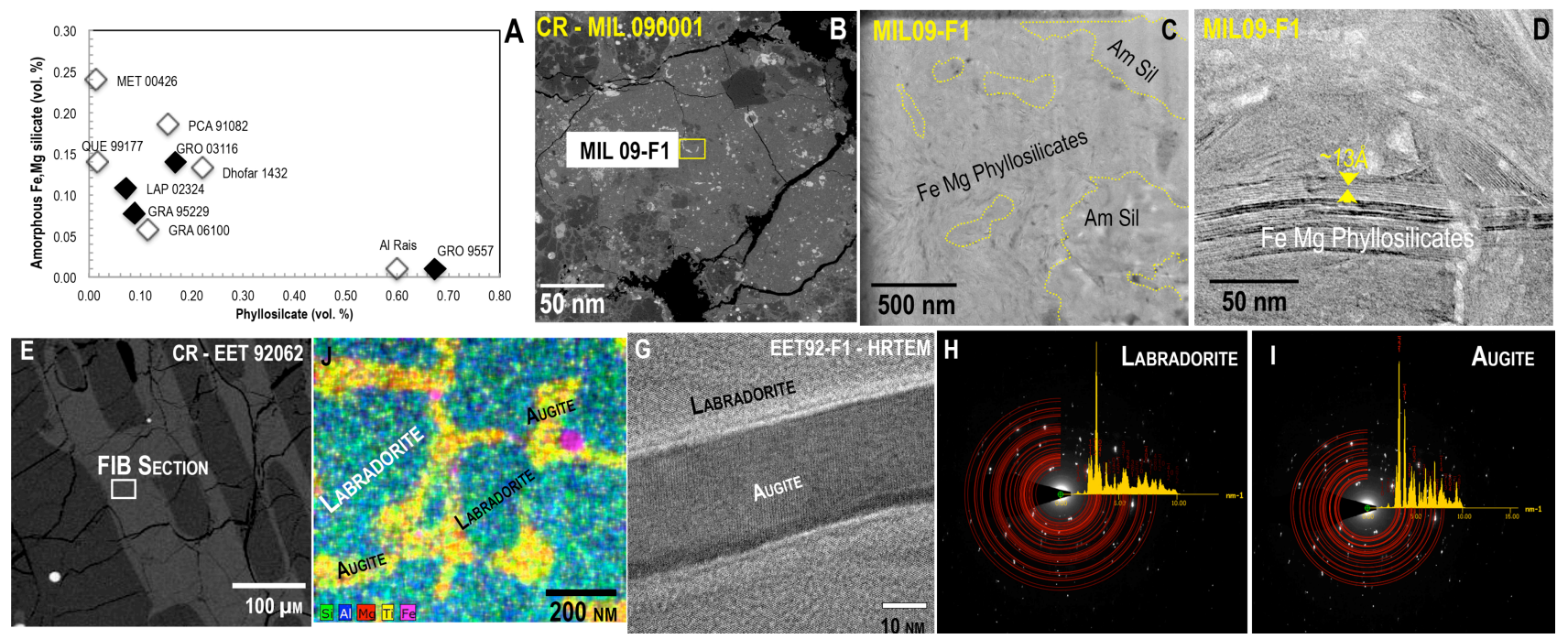

Figure 1. (a) Bulk abundance of amorphous Fe,Mg silicate vs. phyllosilicate determined by PSD-XRD. (b) BSE of region where FIB section MIL09-F1 was retrieved. (c-d) TEM shows that Fe-Mg silicate (amorphous and phyllosilicates) is located in matrix, comprising 30 vol. \% of CRs. (e) BSE image of the type I chondrule mesostasis from which FIB section EET92-F1 was extracted. (f) Overlay X-ray compositional map from EET92-F1, showing the presence of equigranular, labradorite and augite. (g) HRTEM close-up of minerals in (f). (h) Labradorite diffraction pattern. (i) Augite diffraction pattern. 